Running Head: CONFIGURAL LEARNING IN CONTEXTUAL CUING

\title{
Configural learning in contextual cuing of visual search
}

\author{
Tom Beesley ${ }^{1}$, Miguel A. Vadillo ${ }^{2,3}$, Daniel Pearson ${ }^{1} \&$ David R. Shanks ${ }^{2}$ \\ ${ }^{1}$ University of New South Wales, Sydney, Australia \\ ${ }^{2}$ University College London, United Kingdom \\ ${ }^{3}$ King's College London, United Kingdom
}

Mailing address:

Dr Tom Beesley

School of Psychology

University of New South Wales

Matthews Building

Kensington Campus

Sydney, Australia

2052

Tel: +61 (0)2 93853032

e-mail: t.beesley@unsw.edu.au 


\begin{abstract}
Two experiments explore the role of configural representations in contextual cuing of visual search. Repeating patterns of distractors (contexts) were trained incidentally as predictive of the target location. Training these repeating contexts in consistent configurations led to stronger contextual cuing than when contexts were trained in inconsistent configurations. Computational simulations with an elemental associative learning model of contextual cuing demonstrated that purely elemental representations could not account for the results. However, a configural model of associative learning was able to simulate the ordinal pattern of data.
\end{abstract}

Keywords: Attention; Associative learning; Configural learning; Contextual cuing; Computational modeling 
In a typical trial of a visual search task, participants locate a target (e.g., T) amongst a set of distractors (e.g., L's). In studies examining “contextual cuing” of visual search, a given array of distractors repeats intermittently across the course of the experiment, always associated with the same target location. Having experienced a set of such repeating configurations of distractor stimuli, participants' response times (RTs) will typically be faster for locating targets in these repeating configurations in comparison to entirely novel configurations (Chun \& Jiang, 1998). This contextual cuing effect has garnered a great deal of interest from researchers examining the interaction of attention and learning processes. It demonstrates that humans as well as nonhumans (e.g., pigeons: Wasserman, Teng, \& Castro, 2014) are able to incidentally encode aspects of their environment and store them in long-term memory which then guide the future deployment of attention, by decreasing the number of distractors that need to be processed prior to localisation of the target (e.g., Manelis \& Reder, 2012; Tseng \& Li, 2004).

It has been suggested that contextual cuing involves a process of associative learning, whereby the distractors act as predictive cues to the location of the target (Brady \& Chun, 2007; Chun \& Jiang, 1998; Chun \& Turk-Browne, 2008). During the course of training, distractors appear in consistent locations in relation to the target, such that any given distractor can act as a reliable cue to where the target is within that pattern. Since in a typical task all distractors have equal validity, the strengthening of contextual cuing over time may represent not only a strengthening of individual distractor-target associations, but also the acquisition of new, complementary distractor-distractor associations within the pattern (e.g., Beesley, Vadillo, Pearson, \& Shanks, 2015). This paper examines the types of associative representations that form when visual contexts are stored in memory. Specifically we ask whether contextual cuing results in individual, separate, elemental associations between each distractor and the target location, or whether a configural representation of the entire pattern is stored in memory. 
The computational model of contextual cuing put forward by Brady and Chun (2007) proposes that contextual cuing is driven purely by elemental representations. This model takes the form of a simple connectionist network, in which individual stimulus elements (distractors) are represented on the network's input layer, and the target position is predicted by the resulting activation on the output layer. Learning in the model involves the strengthening of associations (connections) between individual distractor locations and a specific target location. These associations modulate the activation that passes forward to target output units; refinement of the associative network - driven by error-correction processes - leads to more accurate (and hence faster) target detection.

Brady and Chun's (2007) elemental model of contextual cuing can be contrasted with a model that forms a configural representation of each presented pattern of distractors. According to models that embody such a configural means of representing stimuli (e.g., Pearce, 1987), the pattern of stimulation as a whole is stored as a unified representation, and it is this configuration of the elements that itself forms an association with the position of the target. The key difference between elemental and configural representations then is the extent to which the co-occurrence of the elements of a pattern will affect responding. For an elemental model, co-occurrence is not particularly important: provided all the elements can be paired individually with the target, it is of no consequence if those elements appear together in varying fashion on each presentation. In contrast, for the configural model the co-occurrence of the elements is crucial: breaking the configuration will weaken the activation of that configural representation and result in weaker learning.

The extent to which contextual cuing is driven by elemental or configural representations was tested in two experiments by Jiang and Wagner (2004). In Experiment 1, participants were presented with repeating search contexts during an initial training phase. The contexts were designed such that they could be broken apart and recombined in unique ways to form new repeated contexts in a final transfer phase. In Jiang and Wagner's design, all elements of the recombined patterns had 
been trained with respect to the same target location in the training phase. The prediction of a purely elemental account of contextual cuing, therefore, is that this process of recombining trained contexts in this transfer phase should have no impact on the ensuing contextual cuing effect. This was indeed the observed finding: performance on recombined contexts was identical to performance on nonrecombined displays (see also Jiang \& Song, 2005; Ogawa \& Kumada, 2008). The results of this experiment are easily handled by Brady and Chun's model, since the model learns only the relationships between individual distractors and target locations. So long as all constituent elements of the pattern maintain their individual relationships with the target stimulus, recombination of the displays in the final phase will not impair the model's performance.

In a second experiment, Jiang and Wagner (2004) trained participants with a series of repeating search patterns, before conducting a final phase in which the patterns were rescaled (the distances between objects were consistently increased or decreased) and displaced (all patterns were moved $4.5^{\circ}$ of visual angle on the screen). Across the transfer phase, participants detected the target more rapidly in repeating patterns they had been trained with compared to novel patterns, despite the displacement and rescaling adjustments. On the surface at least, this result provides evidence for configural learning processes in contextual cuing. In particular, it was argued that rescaling the objects would disrupt “...exactly where each item was with respect to the computer screen and the viewer" (p. 458), but would retain the relationships between the items (the configuration). One problem with this analysis, however, is that it is known that contextual cuing is maintained if the target object is relocated by a small distance within the pattern (Makovski \& Jiang, 2010). One could therefore appeal to an elemental process to explain these data, assuming that the association pertaining to at least one of the distractors (i.e., the distractor closest to the target, see Olson \& Chun, 2002) was able to sustain this adjustment and therefore lead to a generalised response by guiding attention to the target. Brady and Chun (2007) also suggested that this finding is in keeping with their model, since the manipulation retains the within-pattern relationships and only changes the pattern in 
terms of its relative position to the observer. Perhaps more problematic for the interpretation of Jiang and Wagner's data is that it is unclear whether the "transfer effect" simply reflects a phase of new learning; it would not be surprising if some contextual cuing was observed if these transfer patterns were simply learnt anew across the 192 trials in the transfer phase. Their experiment lacks the appropriate comparison trials to adequately assess whether this was the case. Taken together with the results of their Experiment 1, we believe Jiang and Wagner's findings are consistent with an entirely elemental account of contextual cuing.

In contrast, other results cannot be accommodated so easily by purely elemental models of contextual cuing. For instance, a recent series of experiments conducted by Beesley, Vadillo, Pearson, and Shanks (2015) showed that pretraining the associations between the distractors of a search display in an initial phase promoted the ability of those distractor configurations to support contextual cuing at a later stage of the experiment. Since no consistent distractor-target associations were established in this phase (only distractor-distractor associations), these results cannot be explained by elemental models of contextual cuing. In fact, simulations showed that Brady and Chun's (2007) model predicts that pretraining a pattern of distractors in this way would hinder any subsequent learning about those distractor configurations and the target. Beesley et al.'s empirical results therefore suggest that contextual cuing involves learning about the configuration of search displays, such that pretraining a configural representation will facilitate the context-target associations that form during the subsequent contextual cuing phase. Note that this account does not rule out a role for elemental learning processes in contextual cuing. Instead we are suggesting that contextual cuing may rely on the formation of both elemental and configural representations that develop in tandem and provide complementary functions in facilitating search behaviour.

While Beesley et al.'s (2015) results suggest that the acquired associations in contextual cuing cannot be explained by a purely elemental associative learning account, the results are inconsistent with the empirical findings of Jiang and Wagner (2004). Therefore, the present series of experiments 
aims to further explore the configural nature of contextual cuing using a procedure and design closer to that of Jiang and Wagner's (2004) original Experiment 1. One reason why this experiment may have failed to find evidence for configural learning is that the recombined contexts used by Jiang and Wagner were created by combining distractors from only two different training contexts. By recombining patterns in this way, it is possible that sufficient configural information was preserved to produce a contextual cuing effect. It has been shown that localised subsets of repeating context may be sufficient to produce contextual cuing effects equivalent in magnitude to the global context (Olson \& Chun, 2002; Brady \& Chun, 2007). This raises the possibility that greater fractionation of the global context may be required to obtain evidence for configural processes in contextual cuing.

\section{Experiment 1}

In Experiment 1, participants were trained with two sets of repeating patterns. For the consistent set, the arrangement of distractors was such that each pattern was comprised of a unique arrangement of distractor positions (the standard contextual cuing design). For the inconsistent set, the distractors were arranged such that there were fewer co-occurrences of distractors across the course of the experiment. Importantly, the extent to which the individual distractors in the two sets were predictive of the target locations was equivalent. Moreover the inconsistent patterns were broken down into 4 sub-patterns for recombination, rather than just 2, as was the case in the design used by Jiang and Wagner (2004). Thus, Experiment 1 tested the extent to which the consistency of the distractor-distractor pairings influences the development of contextual cuing. A larger cuing effect for the consistent patterns over the inconsistent patterns would provide evidence that configural representations play a role in driving contextual cuing.

\section{Method}

Participants and Apparatus 
The sample size was determined by conducting the experiment for an appropriate number of days to achieve (approximately) a sample size that was twice that (15) used by Jiang and Wagner (2004). A sample of thirty-seven participants was recruited from the University of New South Wales School of Psychology participant pool. Participants took part in exchange for course credit. The experiment lasted for approximately 35 minutes.

\section{Materials and Apparatus}

The experiment was conducted on PCs with 17" TFT monitors set at a resolution of $1024 \mathrm{x}$ 768. Stimulus presentation and response recording were handled by software programmed using MatLab, Cogent 2000 and Cogent Graphics (www.vislab.ucl.ac.uk/cogent.php). The experiment was programmed by T. Beesley. Stimuli were drawn within the experiment software. The letter stimuli were $13 \mathrm{~mm}$ square. For the distractors, the vertical line of the letter L was offset from the end of the horizontal line in order to increase the similarity between distractor and target shapes $(\mathrm{T})$ and therefore increase the difficulty of the visual search task.

Stimuli were arranged in a square grid of 144 evenly spaced cells (12 x 12) which was positioned centrally on the screen and was $240 \mathrm{~mm}$ square. The grid itself was invisible to participants. The fixation cross (displayed centrally before each trial) was $11 \mathrm{~mm}$ square. Responses to the target stimulus were made with keys $\mathrm{z}$ and $\mathrm{m}$ on a standard PC keyboard. The background colour of the screen was grey. Stimuli were coloured blue, red, green or yellow. Distractor stimuli could be oriented by rotating the letter by $0^{\circ}, 90^{\circ}, 180^{\circ}$, or $270^{\circ}$. Target stimuli could be oriented by rotating the letter by $90^{\circ}$ or $270^{\circ}$. The colour and orientation of the distractors were randomly assigned for each pattern, with the constraint that there could be no more than eight distractors of one colour, and at least two distractors of each colour. Repeating elements of patterns maintained the same colour and orientation for distractor stimuli across repetitions. Target colour was also randomly determined and maintained across presentations of the same pattern, but target orientation was determined randomly within each block of trials. 
Design

Patterns consisted of 17 stimuli (16 distractors plus 1 target), with four distractors placed in each quadrant of the pattern. This method of arranging distractors has been used successfully in our laboratory to produce robust contextual cuing effects (Beesley \& Shanks, 2012; Beesley et al., 2015) and we used this method again here as it allowed us to keep the number of distractors equivalent across the quadrants for the manipulations that follow. However, it should be noted that this method does lead to a slightly more crowded target quadrant (5 objects, rather than 4 ) and as such it is possible that it may affect target identification (e.g., Whitney \& Levi, 2011) and hence the magnitude of contextual cuing. For example, crowding may render targets overall more difficult to identify in our procedure compared to a task in which the target is presented amongst a homogenously distributed display. Alternatively, participants might learn that the target is always in the most crowded quadrant, which in turn may make it easier to identify. Future research could profitably explore the effect of crowding on contextual cuing and on the formation of configural representations, for example by comparing the method employed here with one in which additional distractors are randomly placed in other parts of the search array, or in which the target replaces a distractor.

Eight target locations were used in total, two in each quadrant of the screen. Four locations were used in the consistent and four in the inconsistent conditions. These eight target positions were chosen at random from one of five locations within each quadrant that were approximately equidistant from the centre of the screen. Distractors could not appear in these target locations.

Figure 1 depicts the process in which complete patterns of 16 distractor elements were constructed from the combination of four separate sub-patterns, with each of these sub-patterns composed of 4 distractors (one per quadrant). In the consistent condition those 4 sub-patterns were always presented together, while in the inconsistent condition different recombinations of subpatterns were presented in each block. Consistency was varied within-subjects. Table 1 represents 
the recombination process that we followed for each target location. In the consistent condition, subpatterns $\mathrm{A}, \mathrm{B}, \mathrm{C}$, and $\mathrm{D}$ were always paired with one target location. Two other combinations of subpatterns, EFGH and IJKL, were also paired with that target location. These three search displays, $\mathrm{ABCD}, \mathrm{EFGH}$, and IJKL, were presented once in each block of trials. In contrast, in the inconsistent condition, all the sub-patterns that were predictive of the same target location were recombined in different ways in each block of trials (we define a block as the presentation of 12 consistent patterns and 12 inconsistent patterns). The recombination of these sets of elements in inconsistent patterns was constrained such that each possible pair of sets (e.g, A-B, J-D, K-H) occurred exactly 6 times over the course of the experiment. Even though different recombinations could be used within different blocks, all of the inconsistent sub-patterns, A-L, were shown once in each block. As a consequence, all the sub-patterns were trained with their target location exactly the same number of times in the consistent and the inconsistent conditions. That is to say, the elemental associations between each distractor and the target remained constant across the two conditions. However, the specific combinations of sub-patterns were only repeated across blocks in the consistent condition (therefore resulting in a stronger configuration, should such a representation be formed).

As mentioned above, Table 1 represents how patterns were recombined for each target location. Given that four different target locations were used (one per quadrant) in each condition, each block of the experiment comprised 12 search displays in the consistent condition and 12 search displays in the inconsistent condition. Constraining the possible pairs of sets, as described above, led to 27 inconsistent configurations per target position, resulting in a total of 108 inconsistent patterns, and these were presented twice (once in the first 216 trials and once in the second 216). The same number of presentations of the consistent patterns resulted in 432 trials in total. Procedure 
At the start of the experiment participants received instructions detailing the nature of the visual search task. Example displays were presented and participants were shown the correct response for each orientation of the target.

The experiment was sub-divided into 18 blocks of 24 trials (432 trials in total), which allowed for two full presentations of the entire set of inconsistent patterns shown in Table 1. Each block contained 12 consistent and 12 inconsistent patterns and three presentations of each of the eight possible target locations. The order in which the inconsistent patterns were presented was constrained such that the subsets of distractor elements (e.g., A, B, C) were presented as frequently across the entire experiment in both the consistent and inconsistent patterns.

Trials were presented in a random order with the constraint that consecutive trials across adjoining blocks could not present the same repeated pattern. Any given target position could not repeat on consecutive trials. Target orientation (left or right) was determined randomly, but with an equal number of presentations of each orientation every 8 trials.

Each trial commenced with a fixation cross presented in the centre of the screen for $1000 \mathrm{ms,}$ which was then replaced immediately by the stimulus display. Participants searched for the target stimulus and responded with a left or right response depending on its orientation. RTs were recorded from the onset of the pattern. Following a valid response ( $\mathrm{z}$ or $\mathrm{m}$ ) the pattern was removed from the screen. The response-stimulus interval (hereafter RSI) was 1000 ms. If participants made an incorrect response to the target orientation, "ERROR!" appeared in the centre of the screen for 2000 ms, prior to the RSI. A rest-break of 20 seconds was given every 108 trials (splitting the experiment into 4 equal parts). Trials started automatically after these breaks.

We used two different methods to assess the rate of learning about consistent and inconsistent patterns. Firstly, different rates of learning could be observed simply by comparing the RTs on consistent and inconsistent patterns. Secondly, in blocks 8, 9, 16 and 17, the target position was altered from its trained location and placed in the opposite quadrant of the screen (e.g., top-left to 
bottom right). If participants had learned to expect a target in a trained location for any given pattern, then we would expect a marked increase in RTs on these blocks.

\section{Results and Discussion}

Four participants had accuracy scores below $95 \%$ and were removed from further analysis. The mean accuracy of the remaining 33 participants was $98.8 \%$. One participant produced a mean RT more than 2.5 standard deviations greater than the mean of the sample and was removed as an outlier. Trials on which an incorrect response was made and trials immediately after a rest-break were removed from the analysis of RTs. RTs were log transformed and those RTs 2.5 standard deviations above or below the mean RT were removed (on average, these constituted $4.3 \%$ of all trials).

Figure 2A shows the mean natural $\log$ transformed RT (hereafter $\operatorname{logRT}$ ) for both the consistent and inconsistent patterns across the 18 blocks. Blocks 8, 9, 16 and 17 were test blocks in which the target position was switched for each presented pattern. The trends in Figure 2A suggest that search times were, on average, shorter on consistent than inconsistent patterns across the training blocks. A repeated measures ANOVA on the data from the training blocks $(1-7,10-15,18)$ with factors of pattern (consistent vs. inconsistent) and block failed to find a significant main effect of pattern, $F(1,31)=3.03, \eta_{\mathrm{p}}{ }^{2}=.089, p=.092$, but there was a significant main effect of block $F(13$, $403)=51.59, \eta_{\mathrm{p}}^{2}=.63, p<.001$, and a significant interaction between pattern and block, $F(13,403)$ $=2.12, \eta_{\mathrm{p}}{ }^{2}=.064, p=.012$, suggesting that the efficiency of search through consistent patterns improved at a faster rate than those for inconsistent patterns.

The design of Experiment 1 was such that there was, in fact, no difference in the level of consistency for the two sets of patterns until the start of block 2 (in block 1 the two sets were equally consistent). As such, we would not expect any difference in responding within this block and therefore an analysis was conducted on the data from block 2 onwards. This ANOVA found a main 
effect of pattern, $F(1,31)=4.74, \eta_{\mathrm{p}}{ }^{2}=.13, p=.037$, a significant effect of block $F(12,372)=34.44$, $\eta_{\mathrm{p}}^{2}=.53, p<.001$, and a significant interaction between pattern and block, $F(12,372)=1.83, \eta_{\mathrm{p}}{ }^{2}=$ $.056, p=.042$.

It is also clear from Figure 2A that there are increases in RTs during the switch blocks, most noticeably in the latter stage of the experiment. Figure 2B shows learning scores calculated by subtracting the $\operatorname{logRT}$ data for switch blocks ( 8 and 9 for Test $1 ; 16$ and 17 for Test 2 ) from the $\operatorname{logRT}$ data from adjacent blocks ( 7 and 10 for Test $1 ; 15$ and 18 for Test 2). These learning scores therefore reflect the cost associated with switching the target to an unexpected location, and hence the extent of learning for the different types of pattern. A repeated measures ANOVA with factors of pattern (consistent vs. inconsistent) and test (1 vs. 2 ), revealed a significant effect of pattern, $F(1,31)$ $=4.27, \eta_{\mathrm{p}}^{2}=.12, p=.047$, suggesting that more was learnt about consistent than inconsistent patterns, in line with the results from the analysis of the training trials. The main effect of test was not significant, $F(1,31)=1.52, \eta_{\mathrm{p}}^{2}=.047, p=.23$, and there was no evidence for an interaction between these factors, $F<1$. There was evidence of learning about the consistent patterns in both Test $1, t(31)=2.19, d=.38, p=.036$, and Test $2, t(31)=3.91, d=.68, p<.001$, but not for the inconsistent contexts in either Test $1, t<1$, or Test $2, t(31)=1.72, p=.095$. Considering a one-tailed test of significance (i.e., there is no logical reason to expect slower responding on training trials compared to test trials), the latter Test 2 effect suggests that there was some degree of learning for inconsistent patterns in the experiment.

Overall these results are in line with the hypothesis that contextual cuing involves the encoding of configural representations of repeating displays. A comparison of the RTs on the training trials across the experiment revealed a significantly stronger contextual cuing effect for those repeating patterns trained in consistent configurations. Additional evidence for the facilitation of consistent contexts was observed on the "test" trials, in which the target location was switched to the opposite 
quadrant of the screen from that in which it had been trained. On these test trials it was observed that the switch in target location had a greater effect for consistent compared to inconsistent patterns.

\section{Experiment 2}

One of the reasons why the results of the previous experiment depart from the original findings of Jiang and Wagner (2004) may be related to the different levels of inconsistency achieved in the two procedures. Jiang and Wagner made patterns inconsistent by recombining distractors from two previously trained patterns. This procedure might still preserve an important part of the configural information present in the original patterns. In contrast, in our Experiment 1, inconsistent patterns were created by recombining elements from a larger pool of distractors. Note, however, that even in our Experiment 1, inconsistent patterns still conveyed a small amount of configural information. Each of the sub-patterns A-L presented in Table 1 consisted of four individual distractors (one in each quadrant) that were always preserved together. In principle, it is possible to go a step further in the process of breaking down the configural component of each search display. In Experiment 2, the inconsistent patterns were not constructed by recombining sub-patterns of distractors, but by randomly drawing individual items from a pool of distractors. This allowed us to reduce the amount of consistency to minimal levels.

\section{Method}

\section{Participants, Materials, and Apparatus}

Forty three participants were recruited from the University College London participant pool. Participants were paid $£ 4$ in exchange for the participation. The experiment lasted for approximately 35 minutes. Experiment 2 was programmed by M. A. Vadillo and was run at University College London, which introduced some minor differences in the stimuli and apparatus with respect to Experiment 1. The experiment was conducted on PCs with 17" TFT monitors set at a resolution of 
$1280 \times 1024$. The letter stimuli were $14 \mathrm{~mm}$ square. Unlike in Experiment 1, the vertical line of the letter L was not offset from the end of the horizontal line. This led to the visual search task being marginally easier than in Experiment 1 by making distractors less similar to the target. The stimuli formed a $270 \mathrm{~mm}$ invisible square grid. The fixation cross was $8 \mathrm{~mm}$ square. Unlike in Experiment 1, there were no constraints on the number of elements of each color that could appear in a search display. All other details in the procedure were identical to Experiment 1.

\section{Design and Procedure}

Eight target locations were randomly selected for each participant using the same criteria as in Experiment 1. Four of them (one in each quadrant) were used in the consistent condition and four (also one in each quadrant) in the inconsistent condition. Twelve consistent search displays were constructed as in Experiment 1. They each consisted of 16 distractors (4 per quadrant) plus the target. Each target location was used for three different consistent patterns.

Inconsistent patterns were constructed following a different procedure. For each target location, a "super-pattern" with 48 distractors (12 per quadrant) was created. Then, in each training block this super-pattern was split into three different patterns, each of them with 16 distractors (4 per quadrant). Thus, the three patterns for each target position resulted in 12 inconsistent patterns, each with 16 distractors. This equates the number of patterns and number of distractors presented across the consistent and inconsistent sets of patterns. Splitting the super-pattern into the individual inconsistent patterns was achieved by a random process, with the constraint that each of the distractors in the super-pattern had to be used in one and only one of the three 16-distractor patterns presented in a given block. Because this splitting process was repeated anew in every block of trials, the resulting patterns with 16 distractors that were presented to participants in each block were always different from those seen in the previous blocks ${ }^{1}$. However, all the elements of the superpattern were presented once and only once in each block of trials, ensuring that the individual associations between distractors and targets were identical for the consistent and inconsistent 
conditions. The whole experiment comprised 20 blocks of trials, each of them comprising 12 consistent and 12 inconsistent trials. Unlike in Experiment 1, no switch (test) blocks were used in Experiment 2.

\section{Results and Discussion}

Participants responded with a mean accuracy of $98.86 \%$. One participant produced mean RTs more than 2.5 standard deviations greater than the mean of the sample and was removed as an outlier. RTs were filtered using the same procedure of Experiment 1, which removed on average data from $3.4 \%$ of all trials. The average RTs across training blocks are shown in Figure 3. As can be seen, RTs declined faster and reached lower levels in the consistent condition than in the inconsistent one. A repeated measures ANOVA with factors of pattern (consistent vs. inconsistent) and block revealed a significant effect of pattern, $F(1,41)=6.50, \eta_{\mathrm{p}}{ }^{2}=.137, p=.015$, a significant effect of block, $F(19,779)=71.96, \eta_{\mathrm{p}}{ }^{2}=.637, p<.001$, but no interaction effect ${ }^{2}, F<1$. These results confirm that contextual cuing was stronger for patterns in which the distractors were presented in consistent configurations across the course of training.

\section{General Discussion}

Two experiments explored the representations that people form when learning about repeated stimuli in a visual search task. Previously this learning has largely been described as elemental in nature. For example, Brady and Chun's model (2007) formalizes the process of contextual cuing as involving the learning of discrete associations between the spatial position of each element in the visual array and the spatial position of the target. We sought to explore this process in more detail. Two experiments manipulated the configural properties of the repeated displays with which participants were trained. In each experiment distractors were repeated such that each and every one of the distractors presented in repeated patterns was equivalent in terms of how predictive it was of 
the target location with which it was paired. However, the two sets of patterns were different in terms of how consistently the distractor elements were paired together. In consistent patterns the same configuration of distractors was presented on every trial, while in inconsistent patterns the distractors were rearranged across the course of the experiment, resulting in a host of novel configurations (the two experiments differed in the subtle ways in which this inconsistency was manipulated). In both of the experiments, it was observed that the configural nature of the repeated patterns was important for the development of contextual cuing, with more rapid responses to, and hence greater learning about, the consistent patterns.

The results support the findings of Beesley et al. (2015) in so far as they demonstrate that elemental learning processes are insufficient to account for the entirety of what is learnt during contextual cuing. They are however inconsistent with the conclusions of other research (Jiang \& Wagner, 2004, Experiment 1; see also Jiang \& Song, 2005; Ogawa \& Kumada, 2008) in which search was unimpaired in a transfer phase in which trained patterns were recombined into new configurations. We return to this discrepancy later in the General Discussion.

\section{Simulations with three models of contextual cuing}

The rationale for the current experiments was that evidence for the formation of configural representations, such as the data presented here, cannot be accounted for by purely elemental models of contextual cuing. Here we present simulations with three different models of contextual cuing to formally evaluate possible associative mechanisms responsible for the current data. All three connectionist models represent the spatial location of the distractors on the screen across 144 input units and make a prediction regarding the likelihood of the target being in each of those 144 positions across the screen. The models differ in terms of the internal components that drive these predictions. For brevity we only provide a detailed description of the configural model; for details of our implementation of the other two models, see Beesley et al. (2015). 
Brady and Chun (2007): The model learns by separately associating individual distractor locations with the target position. On each trial an error signal, reflecting the discrepancy in the prediction from each output unit, is used to update the weights between each input unit and that particular output unit. The model therefore explains contextual cuing as a process of strengthening weights between the input units for repeating distractor positions and the contingent target location. Brady and Chun emphasised the importance of spatial constraints in the associative learning process of contextual cuing, and this is reflected in the model by modulating the extent to which associations form across the pattern of input: those locations close to the target form stronger associations than those that are further away.

Beesley, Vadillo, Pearson, and Shanks (2015): The model takes as its starting point the Brady and Chun network described above. In addition, the model also features a set of weights connecting each input unit to every other one, which are strengthened according to whether those input units were activated on the same trial. That is, the model encodes the co-occurrence of distractor stimuli in the display. The model uses this process of "auto-association" to produce an internal activation state reflecting what the model perceives in the display, based on its memory of past trials. This model permits some important processes that may influence contextual cuing. Firstly, we have shown that pre-exposing repeated displays (without a consistent target position) in a first phase of the experiment leads to a stronger contextual cuing effect than would otherwise be observed, and we have shown that this effect is anticipated by the Beesley et al. auto-associator model, but not by the Brady and Chun model. Furthermore, the auto-associative process will allow the model to call upon associations related to input units that are not present in the current display. This form of "mediated excitation" is an interesting prediction from the model and seems to play a key role in the predictions regarding the current experiments (see below).

A configural model of contextual cuing: Here we present a new model of contextual cuing that is quite distinct from the "elemental" models described above. The model is largely based on 
Pearce's (2002) configural model of associative learning with adjustments to allow the presentation of the current search arrays. This model incorporates the storage of entire configurations in memory, which independently become associated with the target location. When a pattern of stimuli is presented to the network, the model first initiates a process of pattern matching against each stored configuration in memory. In each case, a similarity vector $(\mathrm{sim})$ is produced, which reflects the similarity between the current pattern and a configuration stored in memory, calculated as:

$$
\operatorname{sim}(\mathrm{P}, \mathrm{C})=\frac{\Sigma(\mathrm{P} \wedge \mathrm{C})^{2}}{\Sigma \mathrm{P} \cdot \Sigma \mathrm{C}}
$$

where $\mathrm{P}$ is the presented pattern, $\mathrm{C}$ is the comparison configural pattern stored in memory, $\Sigma(\mathrm{P} \wedge \mathrm{C})$ is the number of common elements amongst the two patterns, and $\Sigma \mathrm{P}$ and $\Sigma \mathrm{C}$ are the total number of elements in patterns $\mathrm{P}$ and $\mathrm{C}$, respectively. This similarity computation provides values ranging from $\operatorname{sim}=0$ (unique) to $\operatorname{sim}=1$ (identity). If there is no identity match among the comparisons (i.e., sim $\neq 1$ for any configurations), a new configural unit is added to the network and given a similarity value of 1 for pattern $P$.

Responding to a given pattern is determined by multiplying the similarity values of stored configurations to the current pattern with their respective associative connections to potential target locations. Thus, when pattern $\mathrm{P}$ is presented, responding to output location $\mathrm{O}, R_{\mathrm{O}}$, is determined by:

$$
R_{\mathrm{O}}=\sum_{c} \operatorname{sim}(\mathrm{P}, \mathrm{C}) \cdot V_{\mathrm{O}, \mathrm{C}}
$$

where $V_{\mathrm{O}, \mathrm{C}}$ is the associative strength between the configural unit $\mathrm{C}$ and the output unit $\mathrm{O}$. Changes in associative strength are determined by an error correction learning rule:

$$
\Delta V_{\mathrm{O}, \mathrm{C}}=\alpha \cdot\left(\lambda-R_{O}\right)
$$

where $\alpha$ is a learning rate parameter and $\lambda$ reflects a teaching signal for that position in pattern $\mathrm{P}(\lambda=$ 1 if the target is present in that output location, otherwise $\lambda=0$ ). This learning rule therefore uses error-correction to shape the associative strengths in the model, ensuring more accurate target 
location predictions for subsequent presentations of the same (or similar) repeating configurations. Note that whilst configural units that bear some similarity to the input pattern can influence responding, changes in associative strengths are only permitted for the configural unit that reflects the current input pattern (the identity match).

Simulation results: We compare the performance of the three models on the procedure from the simpler design used in Experiment 2 (i.e., without test blocks). Figure 4 shows the simulation results all three models ${ }^{3}$. In each case we have used the activation of the relevant target output unit in determining the efficiency of the model's search; higher target unit activation values reflect more accurate predictions, and so we subtract this value from 1 to mimic the characteristic decreasing response times in the contextual cuing task, as seen in Figures 2 and 3. Figure 4A shows simulations with the Brady and Chun (2007) model. The model learns rapidly about all presented patterns, as shown by the decreasing search efficiency as the activation of the target output unit increases across training. However, the model does not demonstrate a difference in learning about consistent and inconsistent patterns. This result conflicts with the empirical data we have presented: this pattern of simulation results was anticipated given the elemental nature of the associations in the model.

Figure 4B shows simulation results with Beesley et al.'s (2015) auto-associator model. The model shows rapid learning of both sets of patterns, with increases in the activation of the target output unit leading to greater search efficiency emerging across blocks. In addition the model predicts more efficient search within inconsistent compared to consistent patterns - the opposite result to that observed in Experiments 1 and 2. This is likely to be a result of the auto-associative connections between the units present on the input layer (since this is the only substantial difference between the Brady and Chun and the auto-associator models). A key feature of the auto-associative network is its ability to activate units that are not present in the current display. To understand how this might lead to the current pattern of results, consider a case in which two inconsistent patterns 
that share the same target are presented in close proximity. In this instance, the input-output associations will strengthen for the units activated by the first pattern. When the second pattern is presented it will be able to recruit these same input-output associations that have been strengthened from the presentation of the first pattern, provided that the two inconsistent patterns share a set of common input units, connected via the auto-associative layer. By doing so, the second inconsistent pattern is able to activate representations pertaining to elements that are not present in the given display, bolstering conditioned responding. In contrast, due to their non-overlapping set of distractor features, consistent configurations will not benefit from this process of mediated excitation resulting from the process of auto-association. Hence, prior to asymptotic associative strengths, the autoassociator model erroneously predicts a small benefit for search performance on inconsistent patterns.

Figure 4C shows simulations with the Pearce configural model. It is clear that the model learns readily about both the inconsistent and consistent patterns of context but that learning is greater for the consistent patterns. Whilst the model only associates whole configurations with target locations, it is still able to produce a cuing effect for the non-repeating inconsistent patterns of context. This is due to the generalisation of associative strength from a host of previously presented patterns which share some features with the current pattern. Of course, this generalisation is maximal in the case of consistent patterns and therefore the model predicts greater contextual cuing for these patterns over inconsistent patterns.

We examined the performance of Pearce configural model on the procedure of Experiment 1 and that used in Jiang and Wagner's (2004) Experiment 1. Figure 5A presents simulations of the Pearce configural model for the current Experiment 1. It is clear that, while the simulations produce stronger and more consistent data, the results are very similar to those produced in Experiment 1 (Figure 2A). Better search performance (higher target activations) is seen for consistent patterns compared to inconsistent patterns in the training phases. In the test blocks performance is impaired, 
since here the target is repositioned and so the benefit of the learned configurations is lost. The equivalent performance across consistent and inconsistent patterns in test blocks replicates the patterns seen in the empirical data (Figure 2A).

Figure 5B presents simulations of the Pearce configural model with the procedure from Experiment 1 of Jiang and Wagner (2004). Following the learning of the training patterns, the model shows a deficit in performance on patterns that constitute a recombination of elements from two separately trained patterns. These simulation results are inconsistent with the results of Jiang and Wagner, who showed that performance on recombined patterns was unimpaired in this final phase. This raises the question of why Jiang and Wagner failed to observe evidence of configural learning in their experiment, while in Experiments 1 and 2 the consistency of the configurations modulated learning rate. One possible explanation of this discrepancy is that Jiang and Wagner used quite different methods for generating and recombining patterns, and that these procedural differences may have resulted in more or less rapid rates of acquisition across the different experiments. Closer inspection of the simulation results with the Pearce configural model across the three experiments (Figures 4C, 5A and 5B), shows that the rate of acquisition of the repeating patterns is equivalent across the three simulated experiments (i.e., performance is almost identical across blocks 1-7 in the three experiments). This is perhaps not surprising for two reasons. Firstly, the learning rate is held constant across the three experiments, resulting in the formation of configuration-target associations at the same rate in the simulations with all three procedures. Secondly, the Pearce model has no limitation on the number of patterns it can learn in a given procedure. While the number of consistent patterns in Jiang and Wagner's (2004) procedure was higher than in ours (18 compared to 12 in Experiments 1 and 2), the model will readily form independent configural representations for each of these patterns. In addition, given a sufficiently flexible encoding structure for the patterns presented to the model (144 units in our case), the generalisation that might be expected between such a large number of patterns appears to be having little effect in the model. 
The simulation results do not provide an explanation of the discrepancy between our results and those of Jiang and Wagner (2004). It should be noted that there are other procedural differences between the current experiments and Jiang and Wagner's Experiment 1 (such as the possible effect of object crowding in the current procedures, as noted earlier) which could be explored in future work to identify possible explanations for the discrepancy in the results. An alternative explanation of the discrepancy is that the conclusions from Jiang and Wagner's Experiment 1 rely on a null result, namely, the absence of a significant difference in responding to the trained and recombined patterns. The mere fact that an effect reaches statistical significance in one study (the current experiments), but fails to do so in another (e.g., Jiang \& Wagner, 2004), does not necessarily imply that the results of those two experiments were substantially different. We used a random-effects meta-analysis to obtain a clearer insight into the similarities and differences between our results and those of similar experiments revealing null results. We included in the meta-analysis Experiment 1 from Jiang and Wagner (2004), Experiment 1 from Jiang and Song (2005), and the results from the training phases of our two experiments ${ }^{4}$. We did not include the results of the testing phase from Experiment 1 because including data from both the training phase and the testing phase would have violated the independence of the data included in the meta-analysis. However, the choice of one or the other phase does not affect the results of the analysis. As a measure of effect size, we computed Cohen's $d$ scores from the $t$ - and $F$-values reported in these studies (for details of the computation, see Lakens, 2013). The meta-analysis was conducted with the metafor R package (Viechtbauer, 2010).

The results of the meta-analysis are depicted in Figure 6. As can be seen, although the studies conducted by Jiang and Wagner (2004) and Jiang and Song (2005) failed to obtain statistically significant results, the confidence intervals of their effect size estimates overlap considerably with the confidence intervals of the effect sizes in our two experiments. This suggests that, despite their different designs and procedures, the results of all four experiments are actually in reasonable 
agreement and that the fact that some of them reached statistical significance and some of them failed to do so may be attributed to differences in statistical power and precision. Furthermore, the analysis of heterogeneity across studies failed to reach statistical significance, $Q(3)=1.81, p=.613$, suggesting that there is little or no systematic variability across studies. Most importantly, the metaanalytic effect size is significantly different from zero, $z=2.97, p=.0029$, and the confidence interval of the random effects model overlaps with the confidence intervals of each of the four studies. This analysis confirms that there is no fundamental disagreement between the present experiments and previous studies that failed to obtain configural effects at $p<.05$.

To summarize, the different findings presented across these studies are likely to be a result of two factors. Firstly, in the current experiments, more sensitive manipulations of configural information were employed, which we believe led to the observation of effects consistent with configural learning. Secondly, inadequate sample sizes were used in previous studies, which may have resulted in under-powered experiments, and hence null results with regards to the role of configural processes. Given these differences, the collective body of evidence available so far leads to the interpretation that configural representations are formed during contextual cuing.

To what extent is contextual cuing driven only by configural representations of the context? Our current data speak to this question, in as much as participants appeared to learn about the repeating elements of the inconsistent patterns in Experiment 1: there is a contextual cuing effect in the data from Test 2 (significant at $p<.05$, one-tailed). While there was some configural information present in the inconsistent displays, the information was dispersed across just four distractors, with one of these distractors presented in each quadrant of the screen (see Figure 1). It therefore seems unlikely that this learning effect would be driven by the learning of a configuration and it is more likely that the weaker contextual cuing effect we observed in this condition was due to the elemental association between a single distractor and the target (i.e., the closet distractor, see Brady \& Chun, 2007). The implication of this is that contextual cuing may well be the product of both configural and 
elemental representations. Future research could employ the stronger design of Experiment 2, in which configural information was even more diluted, together with an appropriate control condition against which to measure learning of the inconsistent patterns.

Configural models offer an extremely flexible system for storing stimulus representations and have been shown to account for a wealth of data within the field of associative learning (for a review see Pearce, 2002). This flexibility is a result of the generalised response mechanism implemented in Equation 2 above. This aspect of the model ensures that should a presented pattern fail to match a stored pattern in memory, conditioned responding to that pattern can still occur as a result of the similarity between the two patterns. This allows the model to generalise to patterns it has not been trained on in the past, such as a pattern that has been transformed (Jiang \& Wagner, 2004, Experiment 2), a pattern that is recombined with another (Jiang \& Wanger, 2004, Experiment 1), or a pattern in which some of the elements are missing or randomly arranged (Beesley \& Shanks, 2012). Configural models also make unique predictions about the readiness with which certain combinations of stimuli can be learnt. For example, in a "negative patterning" design, when two stimuli are presented independently they predict the occurrence of an outcome $(\mathrm{A}+$ and $\mathrm{B}+)$, but when combined the outcome does not occur (AB-), and many animals (e.g., rats, dogs, humans) are able to solve this discrimination (e.g., Pavlov, 1927; Rescorla, 1973). It is, however, difficult to account for in terms of elemental processes, since any association that forms between the elements and the outcome will lead to a strong response when the two elements are combined (due to a summation of the independent associations). Configural models on the other hand can readily explain the result, since for these models the $\mathrm{AB}$ compound forms a unique representation in memory, and it is this representation that forms an associations with the outcome, independently of the associations that form for the representations of the elements. One could extend such patterning designs to the contextual cuing procedure by training two separate patterns as predictive of the target in location 1 , but when combined (i.e., a larger pattern) the target would be placed in location 2. Evidence of a 
contextual cuing effect for both the individual patterns and their configuration would provide further compelling evidence for configural processes in contextual cuing.

Why might contextual cuing in particular rely on configural processing? The question of when learning processes operate upon elemental or configural representations is not a new one for the field of associative learning (for a review, see Melchers, Shanks, \& Lachnit, 2007). For example, Alvarado and Rudy (1992) were the first to demonstrate that the chosen mode of processing (elemental or configural) is not fixed, but instead can be altered on the basis of past experience. Rats trained with a discrimination that requires a configural solution will subsequently adopt a similar configural solution to new discriminations that can also be solved elementally. Of particular relevance to the present experiments are the effects that different stimulus properties have on the formation of configural representations. For example, it is usually seen that when elements of a stimulus belong to the same modality (e.g., visual), configural processing is more likely to be observed (in comparison to multisensory stimuli) (e.g., Keyhoe, Horne, Horne, \& Macrae, 1994). In addition, the organization of stimuli is known to affect the way they are grouped into configurations during human causal learning tasks. For example, Glautier (2002) has shown that when two cues are presented in a proximal grouping, then less competition occurs between the two elements (they are treated as a whole configuration). Thus, on the basis of these findings, the close proximity of cues within the same modality in the contextual cuing task would be expected to result in configural processing of the display. Previous data have shown that the proximity of distractors to the target is imperative for strong contextual cuing effects (e.g., Olson \& Chun, 2002; Brady \& Chun, 2007). Similarly, it might be expected that the spacing of the distractors across the display could determine the extent to which they form configural units, or conversely the extent to which they compete for associative strength in an elemental fashion (cf. Beesley \& Shanks, 2012).

To date, there has been scant evidence in the contextual cuing literature for the formation of configural representations. We believe that a key reason for this lack of evidence is that the large 
number of elements in a typical visual search display makes it very difficult to isolate the contribution of individual elements to the contextual cuing effect. The present experiments went to great lengths to break this consistency in the repeated patterns, and therefore provides the clearest evidence to date that when patterns of visual search repeat, they necessarily form a configural representation. A computational model of contextual cuing that encodes such a configural representation was shown to provide the best account of the data, while elemental models of contextual cuing cannot account for these effects. While we believe that ultimately contextual cuing may be a result of multiple representational forms (i.e., configural and elemental), these simulations are a starting point for future models that will investigate exactly how these representations combine to control behaviour during visual search. 


\section{Footnotes}

1. While it is not impossible that a participant may have been presented with the same inconsistent pattern twice across the course of the experiment, the large number of permutations ensured that the probability of this event occurring was extremely low.

2. Unlike in Experiment 1 there was no interaction effect between the factors of pattern and block. We believe this is likely to be due to the fact that participants received 20 training blocks of 24 trials in Experiment 2, compared to 14 training blocks of 24 trials in Experiment 1, allowing the interaction effect to become less prominent feature of the data as learning reached an asymptotic level.

3. All three models were run with 100 simulated participants and a learning rate of 0.15 , as well as the following additional parameter settings for the Brady and Chun (2007) and Beesley et al. (2015) models: momentum $=0.80$; bottom-up activation $=.95$; initial variance of $+/-0.05$. To equate the output of the Brady and Chun model with the other models used, the model was run without the fixed weights embodying the spatial constraints. The model makes similar predictions with these weights in place, but with higher target activation values for all patterns.

4. Ogawa and Kumada's (2008) results cannot be included in the meta-analysis, since there was insufficient information in that report to permit a measure of effect size to be computed. Similarly, only the first experiment by Jiang and Song (2005) was conducted with a standard contextual cuing paradigm. 


\section{References}

Alvarado, M. C., \& Rudy, J. W. (1992). Some properties of configural learning: an investigation of the transverse-patterning problem. Journal of Experimental Psychology: Animal Behavior Processes, 18, 145-153.

Beesley, T., \& Shanks, D. R. (2012). Investigating cue competition in contextual cuing of visual search. Journal of Experimental Psychology: Learning, Memory, and Cognition, 38, 709-725.

Beesley, T., Vadillo, M. A., Pearson, D., \& Shanks, D. R. (2015). Pre-exposure of repeated search configurations facilitates subsequent contextual cuing of visual search. Journal of Experimental Psychology: Learning, Memory, and Cognition, 41, 348-362.

Brady, T. F., \& Chun, M. M. (2007). Spatial constraints on learning in visual search: Modeling contextual cuing. Journal of Experimental Psychology: Human Perception and Performance, 33, $798-815$.

Chun, M. M., \& Jiang, Y. (1998). Contextual cueing: Implicit learning and memory of visual context guides spatial attention. Cognitive Psychology, 71, 28-71.

Chun, M. M., \& Turk-Browne, N. B. (2008). Associative learning mechanisms in vision. In S. J. Luck \& A. Hollingworth (Eds.), Visual memory (pp. 209-245). New York: Oxford University Press.

Glautier, S. (2002). Spatial separation of target and competitor cues enhances blocking of human causality judgements. Quarterly Journal of Experimental Psychology, 55B, 121-135.

Jiang, Y, \& Song, J.-H. (2005). Spatial context learning in visual search and change detection. Perception \& Psychophysics, 67, 1128-1139.

Jiang, Y., \& Wagner, L. C. (2004). What is learned in spatial contextual cuing-configuration or individual locations? Perception \& Psychophysics, 66, 454-463. 
Kehoe, E. J., Horne, A. J., Horne, P. S., Macrae, M. (1994). Summation and configuration between and within sensory modalities in classical conditioning of the rabbit. Animal Learning and Behavior, 22, 19-26.

Lakens, D. (2013). Calculating and reporting effect sizes to facilitate cumulative science: A practical primer for t-tests and ANOVAs. Frontiers in Psychology, 4, 863

Makovski, T., \& Jiang, Y. V. (2010). Contextual cost: when a visual-search target is not where it should be. The Quarterly Journal of Experimental Psychology, 63, 216-25.

Manelis, A., \& Reder, L. M. (2012). Procedural learning and associative memory mechanisms contribute to contextual cueing: Evidence from fMRI and eye-tracking. Learning \& Memory, $19,527-534$.

Melchers, K. G., Shanks, D. R., \& Lachnit, H. (2008). Stimulus coding in human associative learning: flexible representations of parts and wholes. Behavioural Processes, 77, 413-27.

Ogawa, H., \& Kumada, T. (2008). The encoding process of nonconfigural information in contextual cuing. Perception \& Psychophysics, 70, 329-336.

Olson, I. R., \& Chun, M. M. (2002). Perceptual constraints on implicit learning of spatial context. Visual Cognition, 9, 273-302.

Pavlov, I. P. (1927). Conditioned reflexes: An investigation of the physiological activity of the cerebral cortex (G. V. Anrep, Trans.). New York: Dover.

Pearce, J. M. (1987). A model for stimulus generalization in Pavlovian conditioning. Psychological Review, 94, 61-73.

Pearce, J. M. (1994). Similarity and discrimination: A selective review and a connectionist model. Psychological Review, 101, 587-607.

Pearce, J. M. (2002). Evaluation and development of a connectionist theory of configural learning. Animal Learning \& Behavior, 30, 73-95. 
Rescorla, R. A. (1973). Evidence for a unique stimulus interpretation of configural conditioning. Journal of Comparative and Physiological Psychology, 85, 331-338.

Tseng, Y.-C., \& Li, C.-S. R. (2004). Oculomotor correlates of context-guided learning in visual search. Perception \& Psychophysics, 66, 1363-78.

Viechtbauer, W. (2010). Conducting meta-analyses in R with the metaphor package. Journal of Statistics Software, 36, 1-48.

Wasserman, E. A., Teng, Y., \& Castro, L. (2014). Pigeons exhibit contextual cueing to both simple and complex backgrounds. Behavioural Processes, 104, 44-52.

Whitney, D., \& Levi, D. M. (2011). Visual crowding: A fundamental limit on conscious perception and object recognition. Trends in Cognitive Sciences, 15, 160-168. 
Configural learning in contextual cuing 31

\section{Author Note}

This work was supported by Grant ES/J007196/1 from the Economic and Social Research Council, awarded to David R. Shanks and Tom Beesley. We thank Brooke Hahn for her assistance in collecting the data for Experiment 1. Correspondence concerning this article should be addressed to Tom Beesley, School of Psychology, University of New South Wales, Sydney, New South Wales 2052, Australia. E-mail: t.beesley@unsw.edu.au. 
Table 1. Schematic of the design of Experiments 1.

\begin{tabular}{|c|c|c|c|c|c|}
\hline \multicolumn{3}{|c|}{ Consistent } & \multicolumn{3}{|c|}{ Inconsistent } \\
\hline \multirow[t]{9}{*}{$\mathrm{ABCD}$} & EFGH & IJKL & $\mathrm{ABCD}$ & EFGH & IJKL \\
\hline & & & $\mathrm{ABGH}$ & EFKL & IJCD \\
\hline & & & ABKL & EFCD & IJGH \\
\hline & & & $\mathrm{AFCH}$ & EJGL & IBKD \\
\hline & & & AFGL & EJKD & $\mathrm{IBCH}$ \\
\hline & & & AFKD & $\mathrm{EJCH}$ & IBGL \\
\hline & & & AJCL & EBGD & IFKH \\
\hline & & & AJGD & EBKH & IFCL \\
\hline & & & AJKH & EBCL & IFGD \\
\hline
\end{tabular}

Note: Letters A-L represent sub-configurations of 4 distractors while complete configurations consisted of an arrangement of 4 sets of 4 distractors (see Figure 1). In consistent patterns, subconfigurations were combined in the same manner across trials. In inconsistent patterns, subconfigurations were combined in different arrangements, yielding many different possible configurations. The table shows one quarter of the full design: participants were presented with 12 consistent configurations and 108 inconsistent configurations. 
Figure 1. A depiction of the procedure for recombining sub-patterns of four elements into complete patterns in Experiment 1.

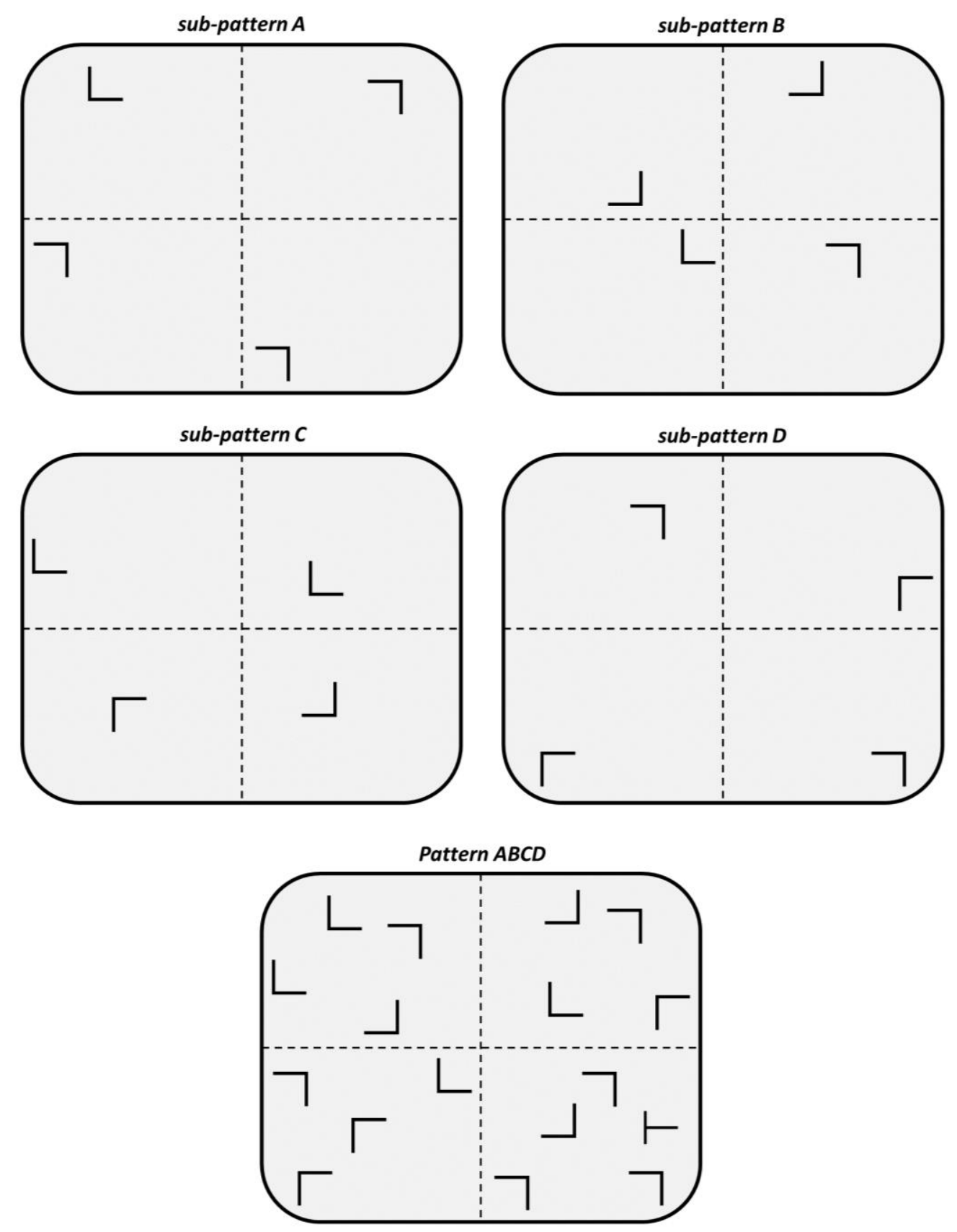


Figure 2. A: Natural $\log$-transformed response time $(\log R T)$ for consistent and inconsistent configurations in Experiment 1. Blocks 8, 9, 16 and 17 represent test blocks in which the target was repositioned in a non-trained location. B: Mean learning scores for test blocks calculated as the difference between $\log \mathrm{RT}$ on test blocks and adjacent blocks (see text for details).

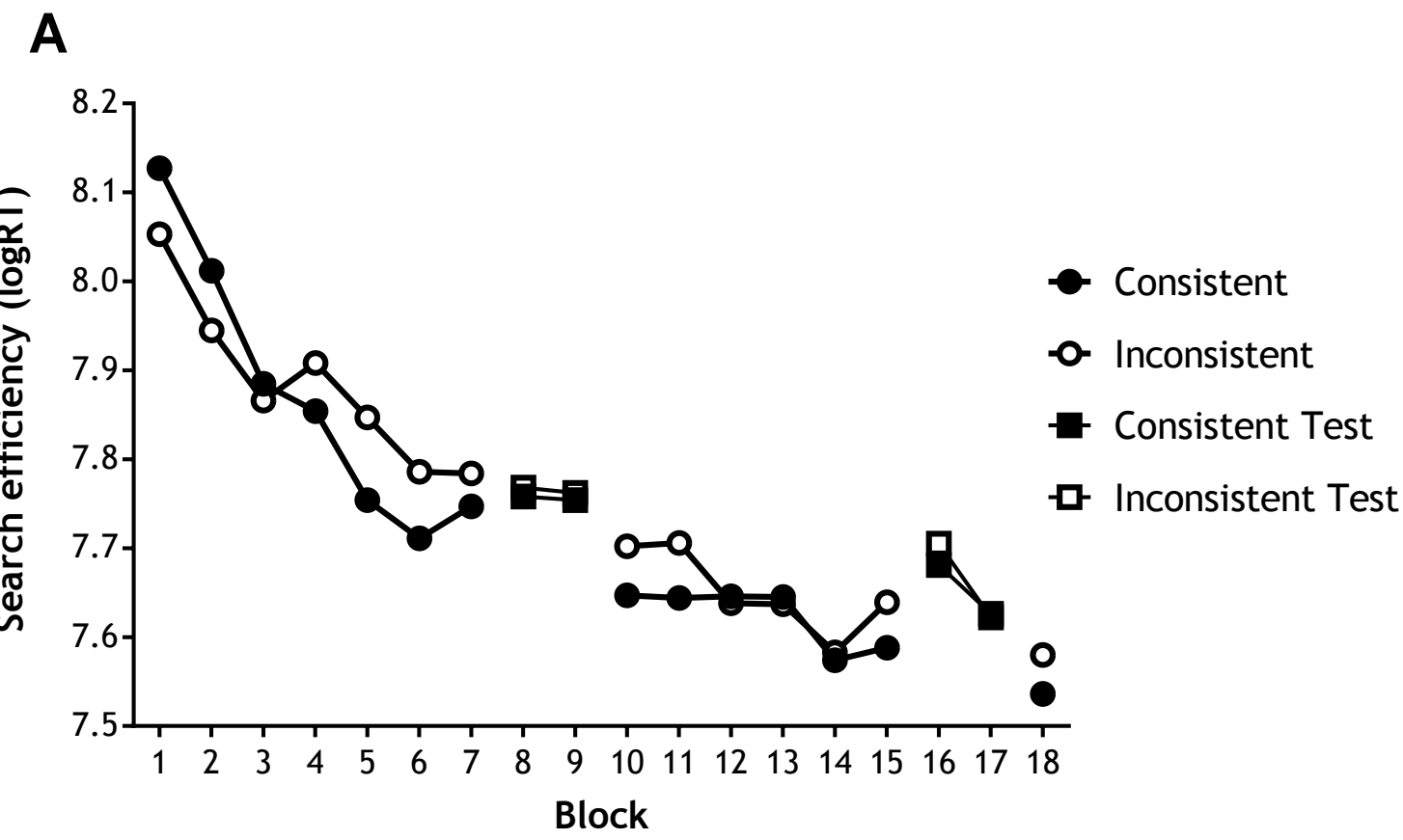

\section{B}

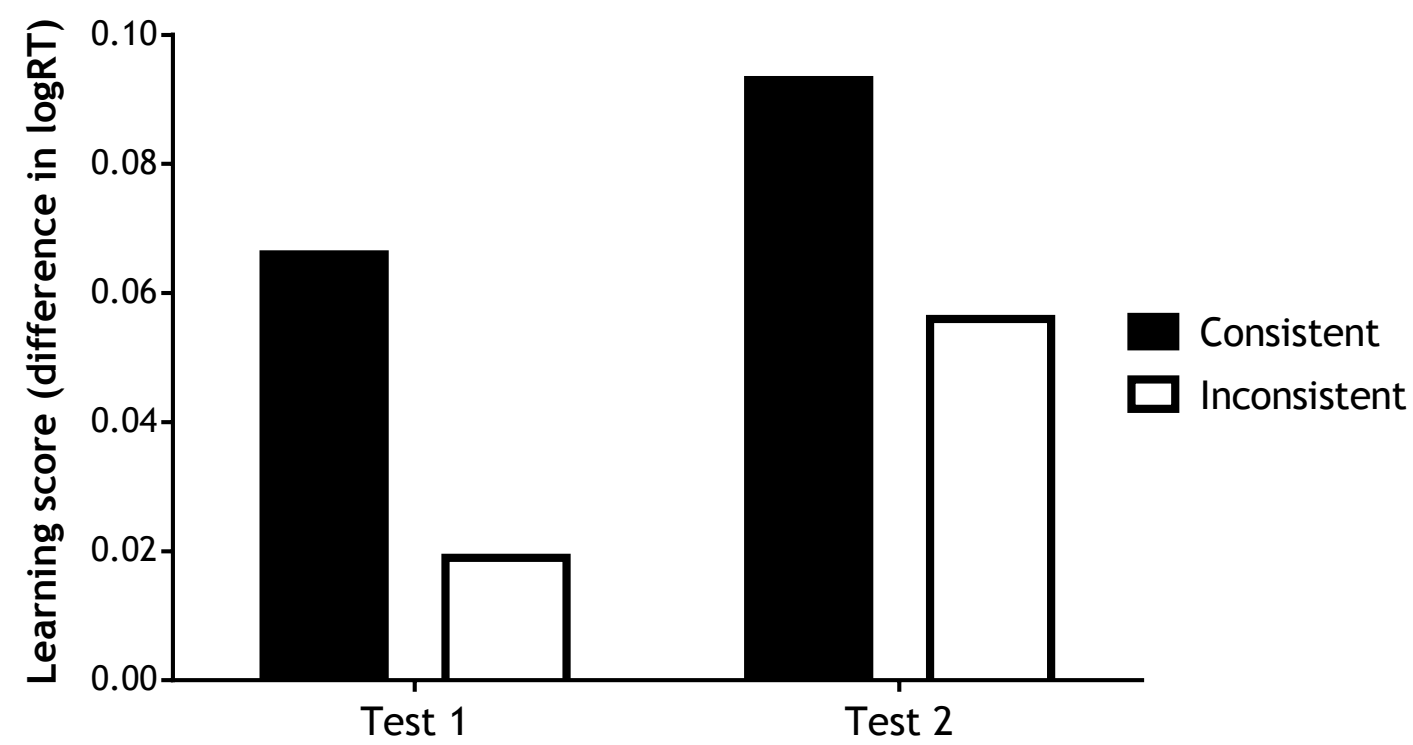


Figure 3. A: Natural log-transformed response time $(\operatorname{logRT})$ for consistent and inconsistent configurations in Experiment 2.

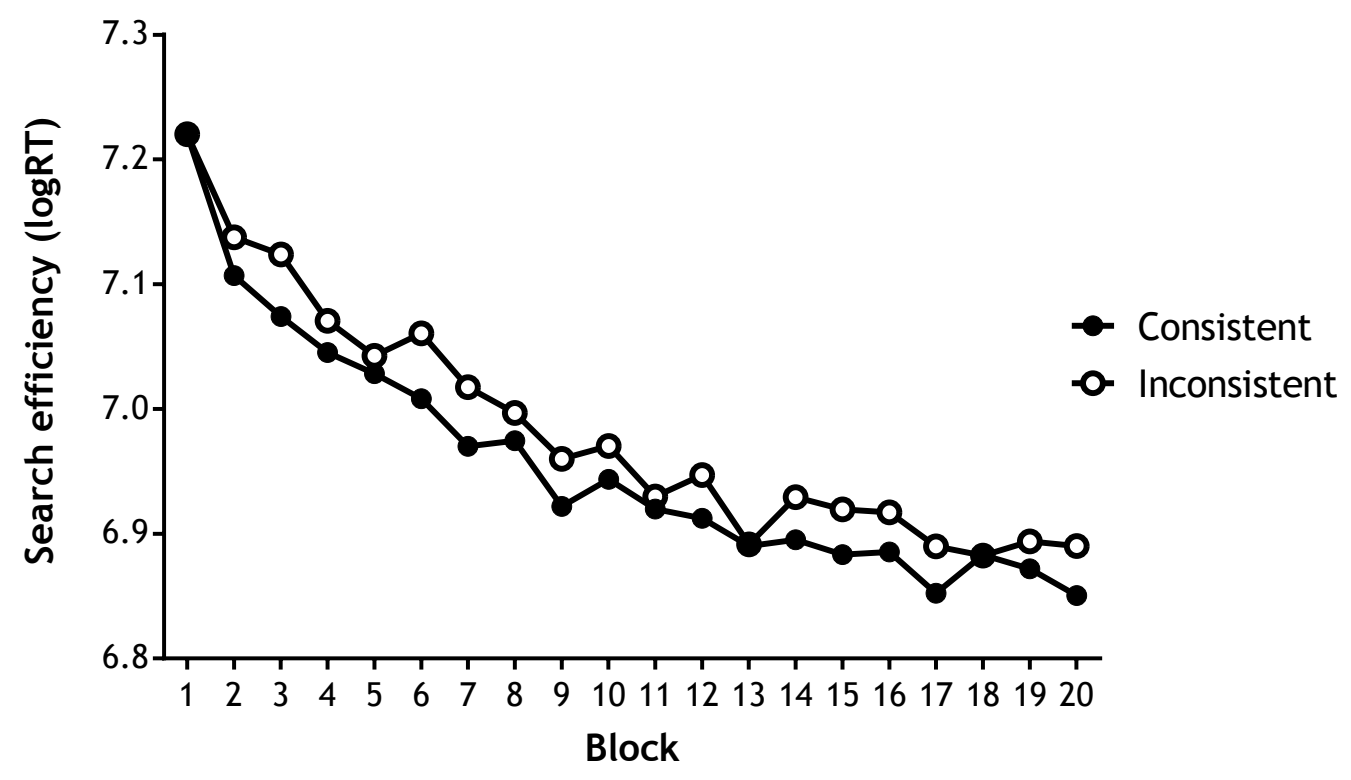


Figure 4. Computational simulation results on the procedure from Experiment 2. A: The elemental model of Brady and Chun (2007); B: the auto-associative elemental model described by Beesley et al. (2015); C: An adapted version of the Pearce (2002) configural model.
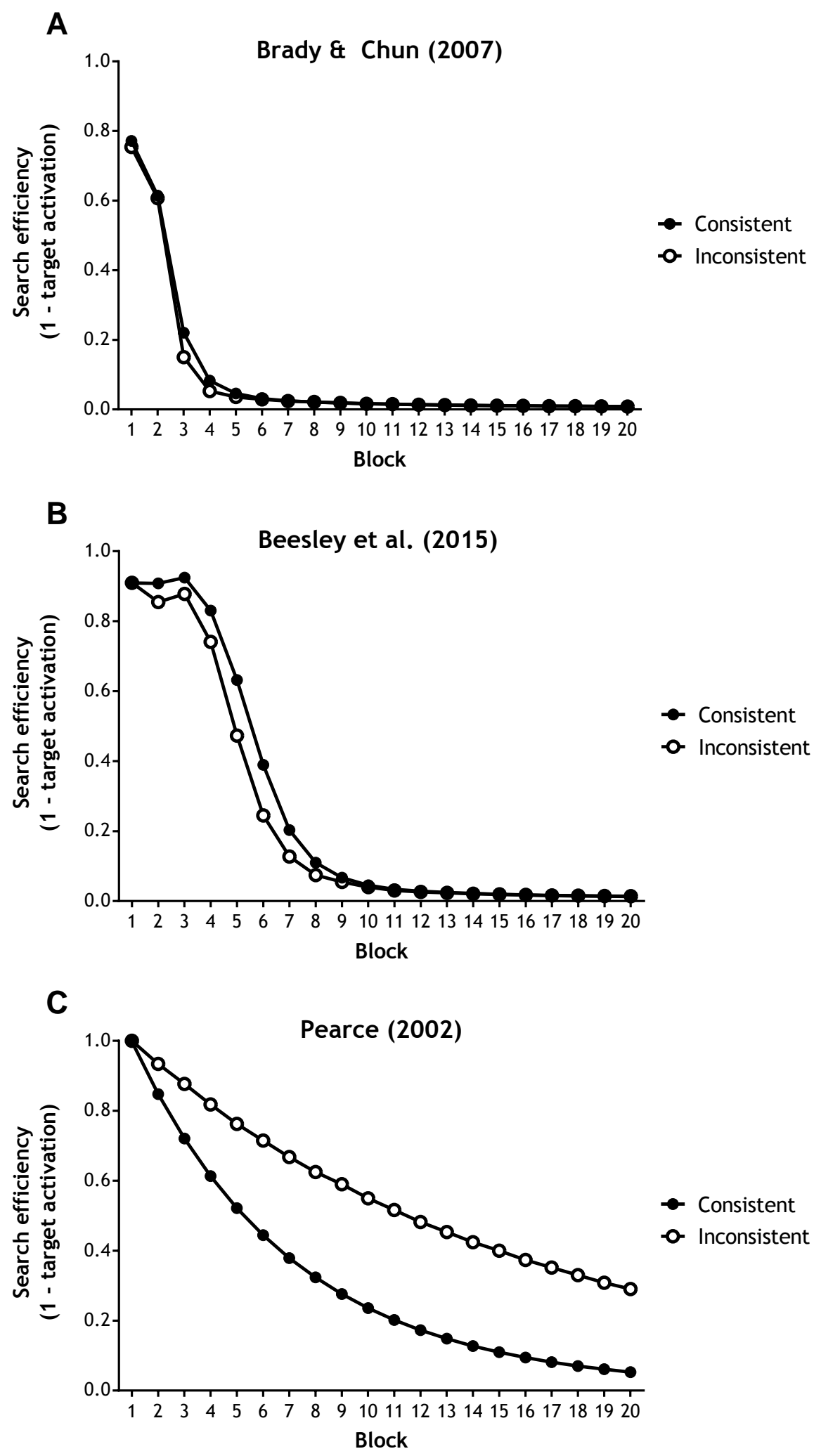
Figure 5. Computational simulations with an adapted version of the Pearce (2002) configural model, conducted on a) the procedure from Experiment 1, and b) the procedure from Experiment 1 of Jiang \& Wagner (2004).
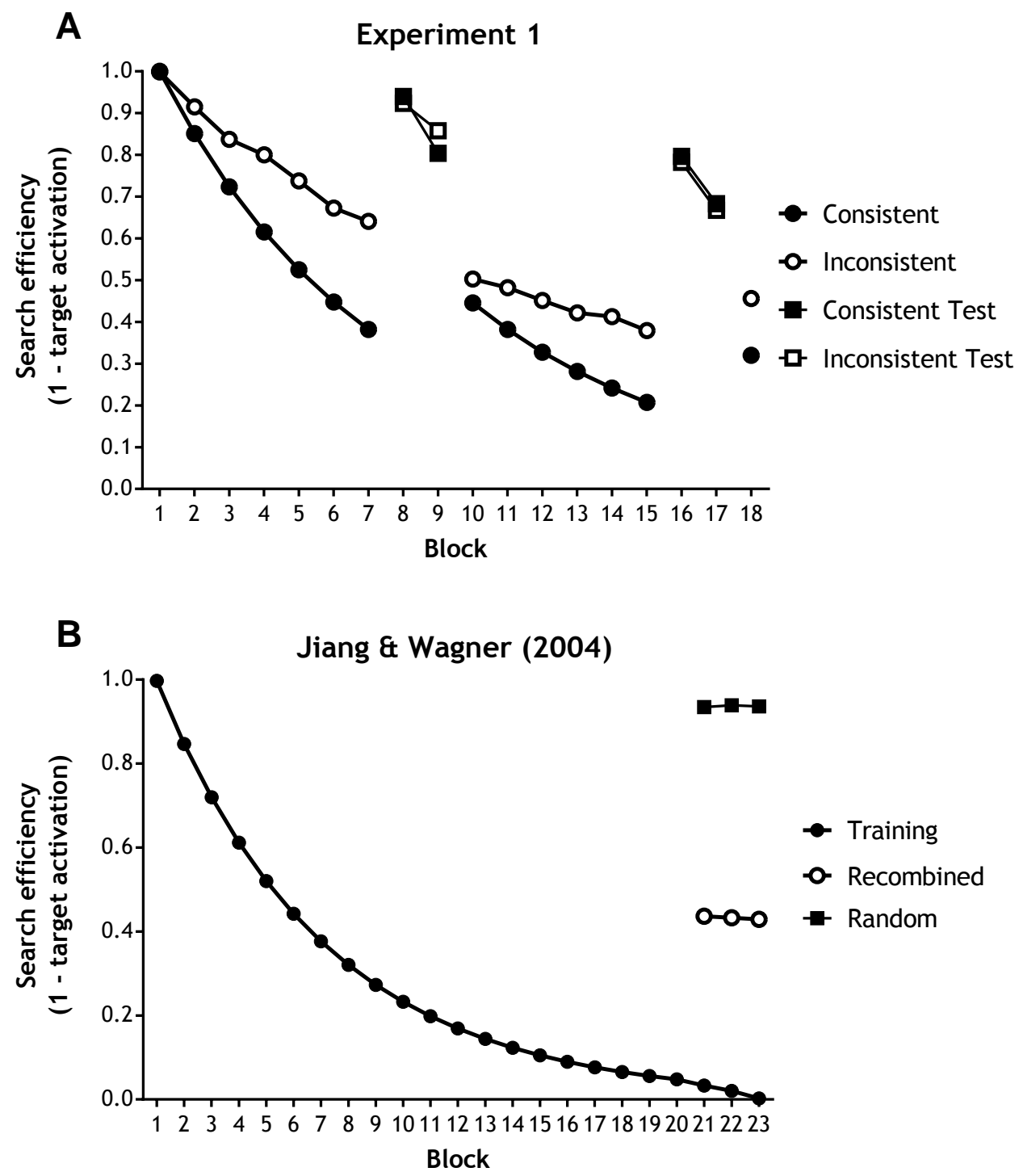
Figure 6. Results of a random-effects meta-analysis including the results of the present experiments and previous studies exploring configural learning in contextual cuing. The size of data points reflects the relative weight of each study in the meta-analytic confidence interval.

\section{Study}

\begin{tabular}{lll}
\hline Jiang \& Wagner (2004, Experiment 1) & $0.09[-0.42,0.59$ ] \\
Jiang \& Song (2005, Experiment 1) & $0.09[-0.45,0.64]$ \\
Experiment 1 & $0.38[0.03,0.74]$ \\
Experiment 2 & $0.39[0.08,0.71]$ \\
Random Effects Model & 0.30 [ $0.10,0.50$ ]
\end{tabular}

ES $\quad 95 \% \mathrm{Cl}$ 